\title{
Influencia de los procesos naturales y antrópicos sobre la calidad del agua en cuatro embalses cubanos
}

\author{
Carmen Betancourt ${ }^{1, *}$, Roberto Suárez ${ }^{2}$ y Fanny Jorge ${ }^{1}$ \\ ${ }^{1}$ Centro de Estudios Ambientales de Cienfuegos. Calle 17, esq. Ave 46 s/n, Reparto Reina. Código Postal 55100, \\ Cienfuegos, Cuba. \\ ${ }^{2}$ Universidad de Cienfuegos. Carretera a Rodas km 3. Código Postal 59430, Cienfuegos, Cuba. \\ * Corresponding author: carmen@gestion.ceac.cu
}

Received: 19/3/2010

Accepted: 23/1/2012

\begin{abstract}
Influence of natural and antropic processes on the water quality in four cuban reservoirs

The waters of the reservoirs Paso Bonito, Avilés, Abreus and El Salto, located at south-center of Cuba, are used in agriculture, fishing, industries and human consumption. The aim of this research was to identify the physicochemical characteristics of the water of these reservoirs and the influential processes. Application of Gibbs and Piper diagrams to the major ions of the water, measured during the period 1986-2005, identified weathering as the natural process with greater influence on chemical composition of the water. The concentrations of major ions were only significantly different between periods (dry and wet) in Abreus reservoir, in relationship to its position within basin. Significant similarities according to ANOVA were frequent between Abreus and El Salto, where the alkalinity was also higher by silicates weathering in correspondence with its geology. The influence of anthropogenic activities on water quality was revealed by mean of $\mathrm{Cl}^{-}: \mathrm{Na}^{+}+\mathrm{K}^{+}, \mathrm{Ca}^{2+}+\mathrm{Mg}^{2+}: \mathrm{Na}^{+}+\mathrm{K}^{+}$ ratios and trophic state index determined by measurements of transparency, nutrients and chlorophyll $a$. Eutrophy was predominant in reservoirs, where the basins are more antropized. The application of a graphical method revealed that values of Chlorophyll a were the cause of turbidity of water in Aviles, Abreus and El Salto and that nitrogen was the limiting nutrient for phytoplankton growth.
\end{abstract}

Key words: Major ions, rock weathering, reservoir, eutrophication.

\section{RESUMEN}

\section{Influencia de los procesos naturales y antrópicos sobre la calidad del agua en cuatro embalses cubanos}

Las aguas de los embalses Paso Bonito, Avilés, Abreus y El Salto, situados en el centro-sur de Cuba, son usadas en la agricultura, la pesca, la industria y para el consumo humano. El objetivo de esta investigación fue identificar las características físicas y químicas de las aguas de estos embalses y los procesos influyentes. La aplicación de los diagramas de Gibbs y trilineal, sobre los componentes mayoritarios del agua medidos durante la etapa 1986-2005, permitió identificar la meteorización como el proceso natural de mayor influencia sobre la composición química de las aguas. Las concentraciones de los iones mayoritarios solo registraron diferencias significativas entre periodos (seco y lluvioso) en el embalse Abreus, lo cual se relacionó con su posición dentro de la cuenca. Las similitudes significativas según el ANOVA, fueron frecuentes entre los embalses Abreus y El Salto, donde también fue mayor la alcalinidad por la disolución de los silicatos, en concordancia con su geología. La influencia de las actividades antrópicas sobre la calidad del agua se reveló mediante la razones $\mathrm{Cl}^{-}: \mathrm{Na}^{+}+\mathrm{K}^{+}, \mathrm{Ca}^{2+}+\mathrm{Mg}^{2+}$ : $\mathrm{Na}^{+}+\mathrm{K}^{+}$y el índice del estado trófico, determinado mediante mediciones de la transparencia y la concentración de nutrientes y Clorofila a. La eutrofia fue mayor en los embalses donde las cuencas estaban más antropizadas. La aplicación de un método gráfico reveló que la turbiedad del agua en los embalses Avilés, Abreus y El Salto se debió a los valores de Chlorophyll a y que el nitrógeno fue el nutriente que limitó el desarrollo fitoplanctónico.

Palabras clave: Iones mayoritarios, meteorización, embalses, eutrofización. 


\section{INTRODUCCIÓN}

Los embalses son considerados centinelas, integradores y reguladores del cambio climático (Williamson et al., 2009). Sin embargo, es necesario disponer de un conocimiento básico sobre la calidad de las aguas embalsadas que permita distinguir el efecto del cambio climático de otros procesos naturales o antrópicos influyentes.

El estudio de los componentes mayoritarios del agua permite reconocer procesos de interacción entre la roca y el agua que son responsables de su composición química (Li et al., 2009). El proceso de meteorización se puede identificar mediante el modelo de Gibbs (1970), el diagrama de Piper (1944) y mediante el uso de relaciones iónicas (Chung et al., 2009). Este proceso es dependiente de la temperatura, de las precipitaciones y de las actividades humanas desarrolladas en las cuencas (Subramanian et al., 2006).

Las actividades antrópicas generadoras de altas cargas de nutrientes estimulan procesos de eutrofización de las aguas. Las aguas eutróficas generan altos costes de tratamiento (Dodds, 2007), así como alteraciones en el olor y el sabor del agua (Peter,
2008) con peligros potenciales para la salud, debido a la presencia de toxinas producidas por algunas especies de cianobacterias (Sömek et al., 2008).

Esta investigación está dirigida a identificar las características físicas y químicas de las aguas de los embalses Paso Bonito, Avilés, Abreus y El Salto situados en cuatro cuencas de la región central cubana, y los procesos que las determinan, a partir del trabajo de campo y del análisis de los datos recogidos en un periodo de 20 años.

Estos resultados constituyen una referencia para la observación de cambios en los procesos geoquímicos influyentes en la calidad química de las aguas en esta región, y representan una herramienta para la gestión del recurso.

\section{MATERIALES Y MÉTODOS}

\section{Área de estudio}

Los embalses Paso Bonito, Avilés, Abreus y El Salto se localizan en la provincia de Cienfuegos, en el centro-sur de Cuba (Fig. 1). Entre los principales usos de sus aguas destacan la agricultura,

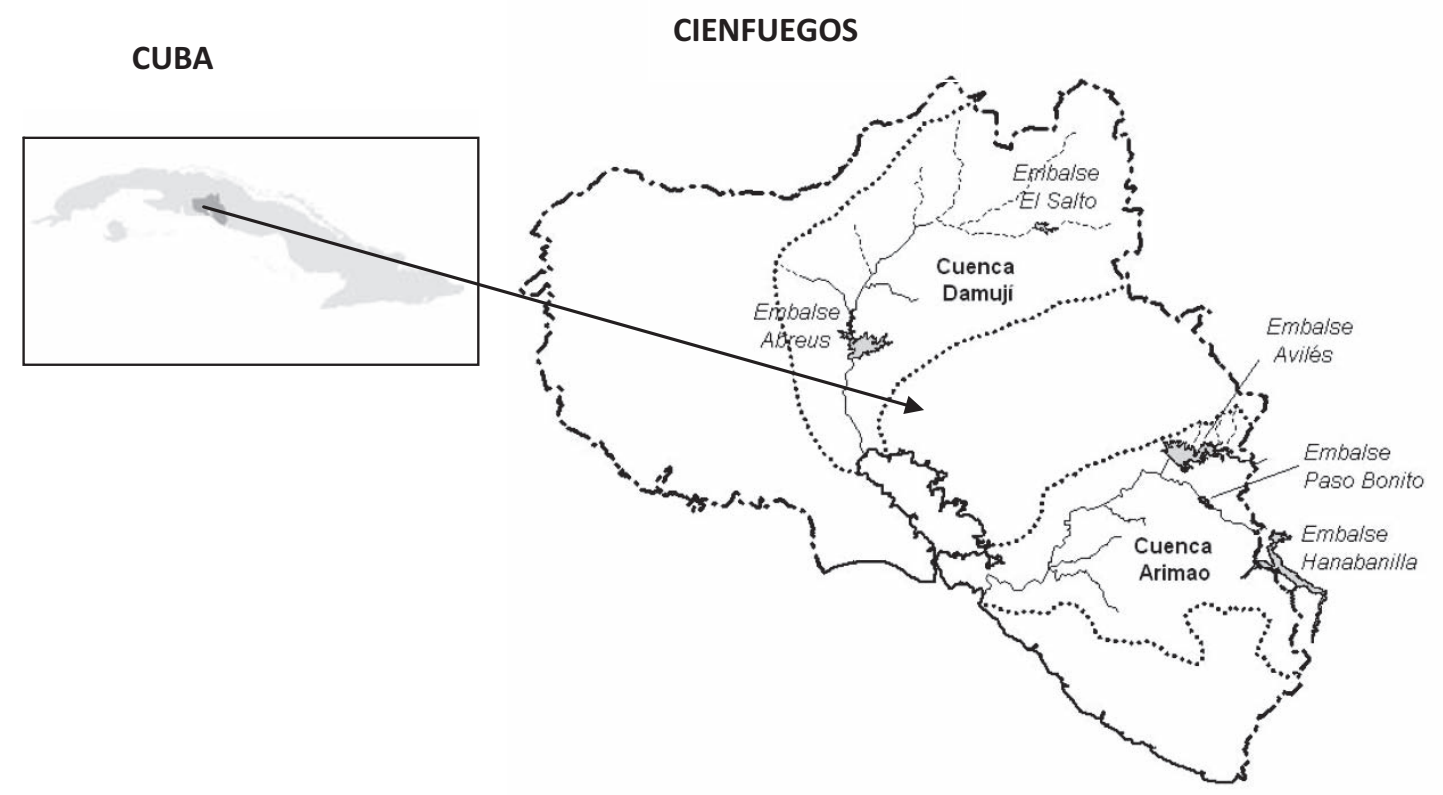

Figura 1. Área de estudio. Se muestran las cuencas de los ríos Arimao y Damují y los embalses Paso Bonito, Avilés, Abreus y El Salto. Study area. The map shows the watershed of Arimao and Damuji rivers and the reservoirs Paso Bonito, Avilés, Abreus and El Salto. 
la pesca y el consumo industrial y humano. Estos embalses son impactados por industrias azucareras y residuos humanos y agrícolas. En las cuencas de los embalses Abreus y El Salto se localiza un mayor número de fuentes de contaminación.

Avilés y Paso Bonito fueron construidos en la parte alta de la cuenca del río Arimao, caracterizado por una topografía variable y una geología compleja, compuesta fundamentalmente por rocas ígneas y metamórficas. En Avilés predominan las llanuras y las rocas anfibolitas, granito, cuarzodioritas con algunas rocas sedimentarias como las calizas, margas y tobas. Paso Bonito tiene su cuenca en la zona montañosa, donde predominan los esquistos.

Los embalses El Salto y Abreus ocupan una llanura en la cuenca del río Damují (en la cabecera y final de la cuenca, respectivamente). Yacen principalmente sobre rocas sedimentarias (margas, calizas, aleurolitas, brechas, conglomerados, areniscas). Las rocas ígneas, en menor cuantía, están representadas por basaltos. Algunas características de los embalses se muestran en la Tabla 1.

\section{Características físicas y químicas}

La base de datos para el estudio de los componentes mayoritarios del agua procede de la Empresa de Aprovechamiento Hidráulico de Cienfuegos. Incluye la conductividad eléctrica (CE), la dureza total $\left(D_{T}\right)$ y la concentración de los iones $\left(\mathrm{HCO}_{3}\right)^{-},(\mathrm{Cl})^{-},\left(\mathrm{SO}_{4}\right)^{2-}, \mathrm{Ca}^{2+}, \mathrm{Mg}^{2+} \mathrm{y}$ $\left(\mathrm{Na}^{+}+\mathrm{K}^{+}\right)$, medidos durante la etapa 1986-2005. Las sales solubles totales (SST) se calcularon mediante la suma de las concentraciones $(\mathrm{mg} / \mathrm{L})$ de los iones antes mencionados.
Para evaluar el estado trófico de los embalses Avilés, Abreus y El Salto se muestreó la superficie del punto de toma durante los meses de abril, agosto, octubre y diciembre de 2008 y marzo de 2009. Se determinó la concentración de fósforo total (TP) por reducción con ácido ascórbico (APHA, 1998), la transparencia del agua como profundidad de visión de un disco de Secchi (SD) de $20 \mathrm{~cm}$ de diámetro, la concentración de clorofila $a$ (CHL $a$ ) por el método de fluorescencia APHA (1998) y la concentración de nitrógeno inorgánico total $\left(\mathrm{Ni}_{T}\right)$ mediante la suma de las concentraciones de nitrógeno amoniacal (N$\left.\mathrm{NH}_{4}\right)$, nitrógeno en forma de nitrito $\left(\mathrm{N}-\mathrm{NO}_{2}\right)$ y nitrógeno en forma de nitrato $\left(\mathrm{N}-\mathrm{NO}_{3}\right)$. Las concentraciones de $\left(\mathrm{N}-\mathrm{NH}_{4}\right)$ se determinaron por formación de indofenol azul, el $\left(\mathrm{N}-\mathrm{NO}_{2}\right)$ por diazotación con sulfanilamida y el $\left(\mathrm{N}-\mathrm{NO}_{3}\right)$ por reducción con hidracina APHA (1998). Estos ensayos se realizaron en el laboratorio del Centro de Estudios Ambientales de Cienfuegos. Los detalles del estado trófico del embalse Paso Bonito fueron evaluados por Betancourt (2009).

\section{Tratamiento de datos}

Para la clasificación del agua según los valores de la CE se usó el criterio de González (2000). Este autor considera que el agua es "ligeramente salina" cuando los valores de la conductividad eléctrica se encuentran en el intervalo 210$400 \mu \mathrm{S} / \mathrm{cm}$ y "medianamente salina" para el intervalo 410-600 $\mu \mathrm{S} / \mathrm{cm}$. La clasificación del agua según su contenido de dureza se realizó de acuerdo con el criterio de Durfor y Becker (1964). Estos autores plantean que cuando la

Tabla 1. Características morfométricas de los embalses. Morphometric characteristics of the reservoirs.

\begin{tabular}{lcccc}
\hline Características* & Paso Bonito & Avilés & Abreus & El Salto \\
\hline Puesta en funcionamiento & 1975 & 1980 & 1986 & 1975 \\
Altitud del embalse $(\mathrm{msnm})$ & 86.3 & 77 & 10 & 52 \\
Altitud media de la cuenca $(\mathrm{msnm})$ & 187 & 165 & 50 & 76 \\
Área de la cuenca $\left(\mathrm{km}^{2}\right)$ & 65 & 310 & 1075 & 80.0 \\
Área del embalse $\left(\mathrm{km}^{2}\right)$ & 1.25 & 77.0 & 5.40 & 2.46 \\
Área de la cuenca: Área del embalse (Ac:Ae) & 52 & 4 & 199 & 32.5 \\
Volumen de almacenamiento $\left(\mathrm{hm}^{3}\right)$ & 8 & 190 & 50 & 9.5 \\
Profundidad máxima del embalse $(\mathrm{m})$ & 19.5 & 36.6 & 12.5 & 17 \\
\hline
\end{tabular}

* Datos tomados del archivo de la Delegación de Recursos Hidráulicos en Cienfuegos. 
dureza total está dentro del intervalo 121-180 $\mathrm{mg} / \mathrm{L}$ el agua se considera "dura". Para valores superiores se clasifica como "muy dura".

Para identificar los mecanismos que intervinieron en la química del agua de los cuatro embalses en estudio se aplicaron las relaciones iónicas y los diagramas de Gibbs (1970) y de Piper (1944), también conocido como diagrama trilineal.

Se aplicó ANOVA de un factor para comparar la calidad del agua referida a los componentes mayoritarios, en los cuatro embalses estudiados. La prueba $T$ para dos muestras independientes se usó para comparar los valores de las razones iónicas y los componentes mayoritarios del agua durante los periodos seco y lluvioso, en los cuatro embalses. Se usó el coeficiente de Spearman para determinar las relaciones entre los índices de estado trófico calculados.

La clasificación trófica se hizo mediante un índice de estado trófico (TSI) sugerido por Carlson (1977) y modificado por Toledo et al. (1983) para zonas tropicales. Las ecuaciones usadas en el cálculo de los índices para las variables diagnóstico (transparencia SD, TP, $\mathrm{PO}_{4}$ y CHL $a$ ) así como el índice de estado trófico medio que combina los índices de cada una de las variables mencionadas se expresan:

$$
\begin{aligned}
& T S I_{\text {Modificado }}(S D)=10 *\left[6-\left(\frac{0.64+\ln (S D)}{\ln 2}\right)\right] \\
& T S I_{\text {Modificado }}(T P)=10 *\left[6-\frac{\ln \left(\frac{80.32}{T P}\right)}{\ln 2}\right] \\
& T S I_{\text {Modificado }}\left(P O_{4}\right)=10 *\left[6-\left(\frac{\ln \left(\frac{21.67}{P O_{4}}\right)}{\ln 2}\right)\right] \\
& T S I_{\text {Modificado }}(C H L a)=\frac{\ln 2}{\quad}=10 *\left[6-\left(\frac{2.04-0.695 \ln C H L a}{\ln }\right)\right]
\end{aligned}
$$

$$
\begin{aligned}
& T S I_{\text {Modificado }}(\text { medio })= \\
& \quad=\frac{T S I(S D)+2\left[T S I(T P)+T S I\left(P_{4}\right)+T S I(C H L a)\right]}{7}
\end{aligned}
$$

Con el resultado del cálculo del $T S I_{\text {Modificado }}$ (medio) se clasificó el estado trófico de las aguas según el siguiente intervalo: Oligotrofia $\leq 44$; Mesotrofia $44<$ TSI $<54$ y Eutrofia $\geq 54$.

Se siguió el criterio propuesto por Morris y Lewis (1988) para evaluar la relación $\mathrm{Ni}_{T}$ :TP (nitrógeno inorgánico total: fósforo total), como indicador del nutriente que limita la productividad del sistema. Estos autores consideran limitaciones de nitrógeno cuando la relación es menor que 0.5, para el intervalo 0.5-4.0, ambos limitan y para valores superiores a 4 es el fósforo el nutriente limitante.

\section{RESULTADOS}

\section{Composición de las aguas y procesos químicos}

Los estadísticos descriptivos de los iones mayoritarios, CE y $D_{T}$ se muestran en la Tabla 2. Los embalses en la cuenca del río Damují (El Salto y Abreus) registraron los valores más altos de la CE, iones mayoritarios y $D_{T}$. En estos embalses el agua fue muy dura según la clasificación de Durfor y Becker (1964), y medianamente salina de acuerdo con la clasificación de González (2000). Según estas clasificaciones, el agua de los embalses construidos en la cuenca del río Arimao (Paso Bonito y Avilés) fue dura y ligeramente salina. En los cuatro embalses las concentraciones de los iones mayoritarios $(\mathrm{mg} / \mathrm{L})$ se pudo ordenar de la siguiente manera: $\mathrm{Ca}^{2+}>$ $\mathrm{Na}^{+}+\mathrm{K}^{+}>\mathrm{Mg}^{2+}$ y $\left(\mathrm{HCO}_{3}\right)^{-}>\mathrm{Cl}^{-}>\mathrm{SO}_{4}^{2-}$ y el agua resultó bicarbonatada cálcica.

La mayor cantidad de similitudes (ANOVA, $p>$ 0.05 ) se encontró entre las concentraciones de los iones mayoritarios de los embalses El Salto y Abreus.

De acuerdo con el diagrama de Gibbs (1970), la composición química de las aguas en los cuatro embalses estuvo controlada por procesos de meteorización. En los embalses construidos en el río Damují las observaciones se localizaron más pró- 
Tabla 2. Estadísticos descriptivos de la conductividad eléctrica, la dureza total y los iones mayoritarios. Descriptive statistics of conductivity, total hardness and major ions.

\begin{tabular}{|c|c|c|c|c|c|c|c|c|c|}
\hline & & $\begin{array}{c}\mathrm{CE} \\
\mu \mathrm{S} / \mathrm{cm}\end{array}$ & $\begin{array}{c}\left(\mathrm{HCO}_{3}\right)^{-} \\
\mathrm{mg} / \mathrm{L}\end{array}$ & $\begin{array}{c}\mathrm{Cl}^{-} \\
\mathrm{mg} / \mathrm{L}\end{array}$ & $\begin{array}{c}\left(\mathrm{SO}_{4}\right)^{2-} \\
\mathrm{mg} / \mathrm{L}\end{array}$ & $\begin{array}{l}\mathrm{Ca}^{2+} \\
\mathrm{mg} / \mathrm{L}\end{array}$ & $\begin{array}{l}\mathrm{Mg}^{2+} \\
\mathrm{mg} / \mathrm{L}\end{array}$ & $\begin{array}{c}\mathrm{Na}^{+}+\mathrm{K}^{+} \\
\mathrm{mg} / \mathrm{L}\end{array}$ & $\begin{array}{c}D_{T} \\
\mathrm{mg} / \mathrm{L}\end{array}$ \\
\hline \multirow{5}{*}{ Paso Bonito } & Media & 257 & 139 & 12 & 7 & 39 & 6 & 7 & 125 \\
\hline & Máx. & 358 & 266 & 25 & 22 & 59 & 19 & 15 & 230 \\
\hline & Mín. & 160 & 70 & 6 & 1 & 13 & 1 & 3 & 65 \\
\hline & DS & 35.8 & 20.1 & 2.5 & 2.7 & 7.6 & 2.8 & 1.9 & 20.2 \\
\hline & $\mathrm{n}$ & 240 & 240 & 240 & 240 & 240 & 240 & 240 & 240 \\
\hline \multirow{5}{*}{ Avilés } & Media & 306 & 147 & 17 & 10 & 29 & 12 & 15 & 125 \\
\hline & Máx. & 377 & 183 & 23 & 17 & 40 & 18 & 21 & 155 \\
\hline & Mín. & 240 & 119 & 12 & 5 & 17 & 7 & 9 & 95 \\
\hline & DS & 30.6 & 13.7 & 2.1 & 2.4 & 4.4 & 2.8 & 2.6 & 12.3 \\
\hline & $\mathrm{n}$ & 105 & 105 & 105 & 105 & 105 & 105 & 105 & 105 \\
\hline \multirow{5}{*}{ Abreus } & Media & 556 & 236 & 41 & 33 & 64 & 13 & 35 & 216 \\
\hline & Máx. & 745 & 327 & 63 & 53 & 94 & 22 & 49 & 318 \\
\hline & Mín. & 380 & 152 & 10 & 19 & 32 & 6 & 16 & 130 \\
\hline & DS & 72.6 & 36.9 & 7.0 & 5.5 & 12.0 & 2.7 & 5.5 & 37.0 \\
\hline & $\mathrm{n}$ & 233 & 233 & 233 & 233 & 233 & 233 & 233 & 233 \\
\hline \multirow{5}{*}{ El Salto } & Media & 479 & 223 & 27 & 23 & 47 & 15 & 32 & 186 \\
\hline & Máx. & 680 & 451 & 46 & 43 & 74 & 31 & 56 & 452 \\
\hline & Mín. & 310 & 147 & 14 & 9 & 21 & 5 & 11 & 90 \\
\hline & DS & 69.0 & 39.5 & 6.1 & 7.0 & 11.2 & 4.9 & 9.1 & 44.1 \\
\hline & $\mathrm{n}$ & 94 & 94 & 94 & 94 & 94 & 94 & 94 & 94 \\
\hline
\end{tabular}

ximas al área de evaporación-cristalización que en los casos de los embalses construidos en el río Arimao, originado por los valores más altos de concentración de SST (Fig. 2). Este resultado es compatible con el obtenido por la aplicación del ANOVA, el cual mostró diferencias significativas $(F=1307 ; p<0.0001)$ en las mediciones de la $\mathrm{CE}$ entre estos embalses. La prueba de comparación múltiple de Tukey reveló que hay diferencias significativas ( $p=0.000$, en todos los casos) al comparar dos a dos.

En el diagrama trilineal los puntos se ubicaron próximos a los vértices que corresponde a los iones $\mathrm{Ca}^{2+}$ y $\left(\mathrm{HCO}_{3}\right)^{-}$en los cuatro embalses (Fig. 3). En los embalses Abreus y El Salto las observaciones se acercaron al área del gráfico que corresponde a las mayores proporciones de $\left(\mathrm{Na}^{+}+\mathrm{K}^{+}\right)$. Este resultado se corroboró con las similitudes (ANOVA, $p>0.05$ ) obtenidas para las concentraciones de $\left(\mathrm{Na}^{+}+\mathrm{K}^{+}\right)$entre dichos embalses y las diferencias $(p<0.05)$ con los embalses Paso Bonito y Avilés.

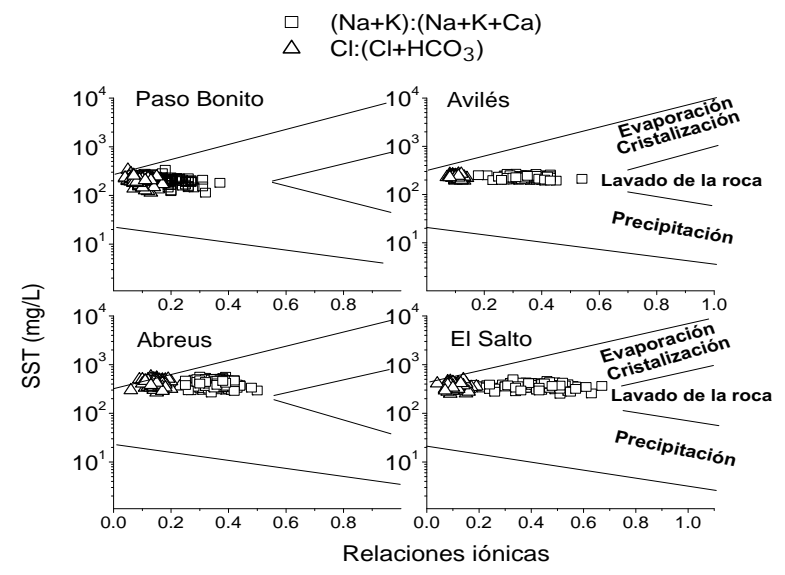

Figura 2. Representación gráfica de la dependencia entre las sales solubles totales y las relaciones iónicas del agua en los cuatro embalses estudiados, según el modelo de Gibbs (1970). La relación SST vs $\mathrm{Cl}^{-}:\left[\mathrm{Cl}^{-}+\left(\mathrm{HCO}_{3}\right)^{-}\right]$se simbolizó con un cuadrado sin relleno y la relación SST vs $\left(\mathrm{Na}^{+}+\mathrm{K}^{+}\right):\left(\mathrm{Na}^{+}+\mathrm{K}^{+}+\mathrm{Ca}^{2+}\right)$ con un triángulo sin relleno. Dependence between total soluble salts and ionic relations of the water for the studied reservoirs: TSS vs $\mathrm{Cl}^{-}:\left[\mathrm{Cl}^{-}+\left(\mathrm{HCO}_{3}\right)^{-}\right]$ and TSS vs $\left(\mathrm{Na}^{+}+\mathrm{K}^{+}\right):\left(\mathrm{Na}^{+}+\mathrm{K}^{+}+\mathrm{Ca}^{2+}\right)$ following Gibbs (1970). SST vs $\mathrm{Cl}^{-}:\left[\mathrm{Cl}^{-}+\left(\mathrm{HCO}_{3}\right)^{-}\right]$is showed by empty square and TSS vs $\left(\mathrm{Na}^{+}+\mathrm{K}^{+}\right):\left(\mathrm{Na}^{+}+\mathrm{K}^{+}+\mathrm{Ca}^{2+}\right)$ by empty triangle. 

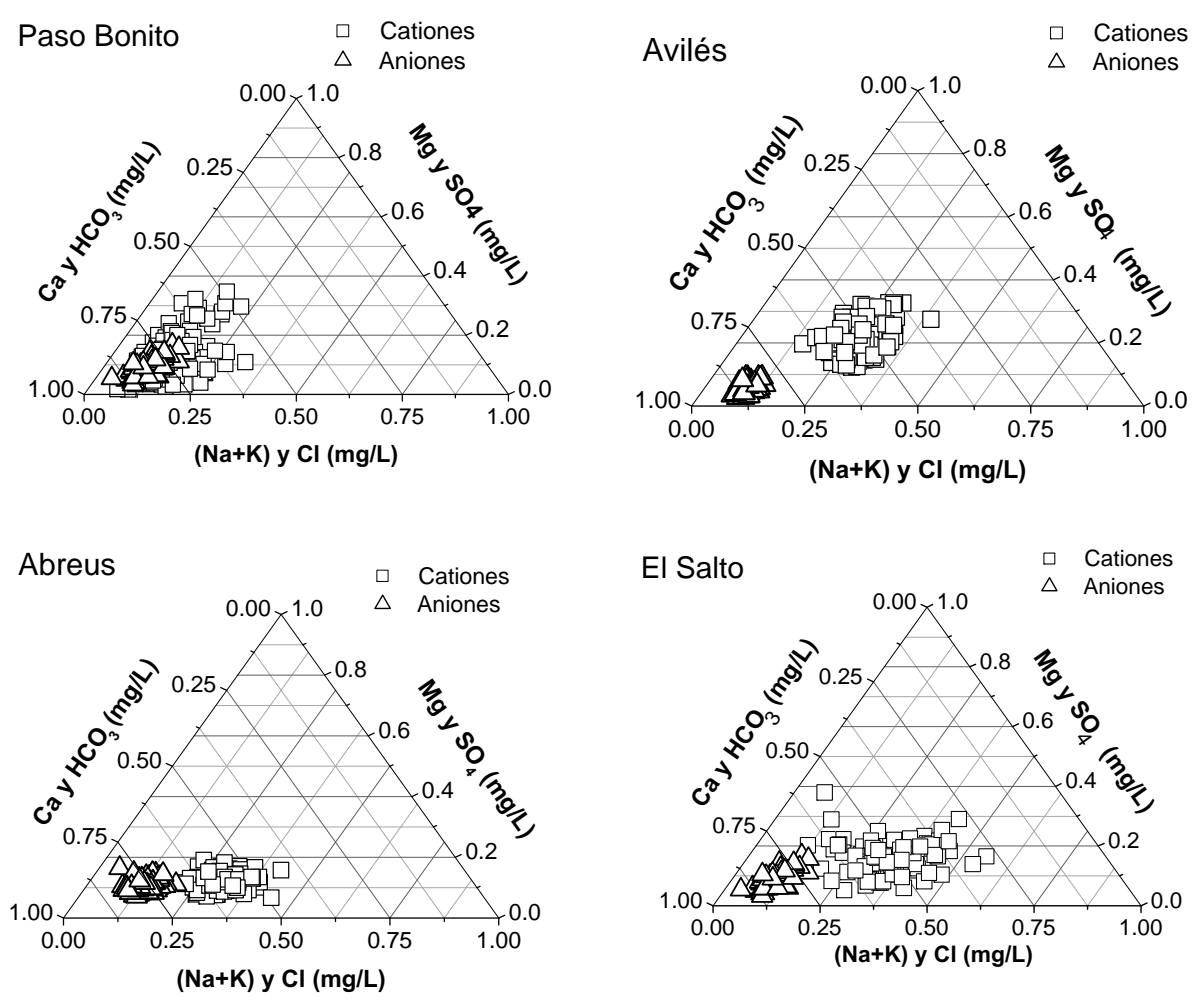

Figura 3. Representación de la composición relativa de aniones (triángulo) y cationes (cuadrado) mediante el diagrama triangular. Muestra la composición relativa de las concentraciones de aniones y cationes expresados en $\mathrm{mg} / \mathrm{L}$ en los embalses situados en la cuenca del río Arimao (Paso Bonito y Avilés) y en la cuenca del río Damují (Abreus y El Salto). Ternary plots for mean concentrations expressed in $\mathrm{mg} / \mathrm{L}$ of anions (triangle) and cations (square) in Paso Bonito and Avilés reservoirs (Arimao watershed) and Abreus y El Salto reservoirs (Damuji watershed).

En el embalse Abreus hubo diferencia significativa $(t=-4.425$ y $p<0.0001)$ para las razones $\left(\mathrm{Ca}^{2+}+\mathrm{Mg}^{2+}\right):\left(\mathrm{Na}^{+}+\mathrm{K}^{+}\right), \mathrm{Ca}^{2+}:\left(\mathrm{SO}_{4}\right)^{2-} \mathrm{y}$ para las concentraciones de los iones mayoritarios ( $p<0.0001)$ al comparar sus valores en ambos periodos. En la figura 4 (C y E) se puede observar que en este embalse las observaciones correspondientes a las razones del periodo seco registraron los mayores valores.

En el embalse El Salto la razón $\left(\mathrm{Ca}^{2+}+\mathrm{Mg}^{2+}\right)$ : $\left(\mathrm{HCO}_{3}\right)^{-}$fue inferior a la unidad (0.99; Fig. 4A), en el resto de los embalses fue superior (entre 1.03 y 1.12) e indicó el predominio de la dureza permanente en sus aguas y la asociación del $\mathrm{Ca}^{2+} \mathrm{y} \mathrm{Mg}^{2+}$ a aniones diferentes al $\left(\mathrm{HCO}_{3}\right)^{-}$.

La elevada contribución del $\mathrm{Ca}^{2+}+\mathrm{Mg}^{2+}$ al total de cationes (TC) en los cuatro embalses se manifestó en los altos valores de la razón $\left(\mathrm{Ca}^{2+}+\mathrm{Mg}^{2+}\right)$ : $\mathrm{TC}$
(Fig. 4B). La mayor contribución se verificó en los embalses Paso Bonito (0.89) y Avilés (0.79).

Las razones $\left(\mathrm{Ca}^{2+}+\mathrm{Mg}^{2+}\right):\left(\mathrm{Na}^{+}+\mathrm{K}^{+}\right)$alcanzaron cifras superiores a la unidad en todos los embalses (en el intervalo 2.85-8.78; Fig. 4C). Los valores más altos corresponden a los embalses situados en la cuenca del río Arimao.

Los valores medios de la razón $\mathrm{Cl}^{-}: \mathrm{Na}^{+}+\mathrm{K}^{+}$ fueron inferiores a la unidad (en el intervalo; 0.590.77), con una distribución de las observaciones por debajo de la línea 1:1, excepto en el embalse Paso Bonito, donde la razón fue 1.21 (Fig. 4D).

En la figura 4E se muestra la poca relación existente entre los iones $\mathrm{Ca}^{2+}$ y $\left(\mathrm{SO}_{4}\right)^{2-}$ con valores de la razón $r=\mathrm{Ca}^{2+}$ : $\left(\mathrm{SO}_{4}\right)^{2-}$ mayores que la unidad.

La alcalinidad producida por la disolución de carbonato (Alcalinidad disolución de carbonato $=0.74$ $\left.\mathrm{Ca}_{\text {total }}+0.4 \mathrm{Mg}_{\text {total }}\right)$ y la producida por la diso- 

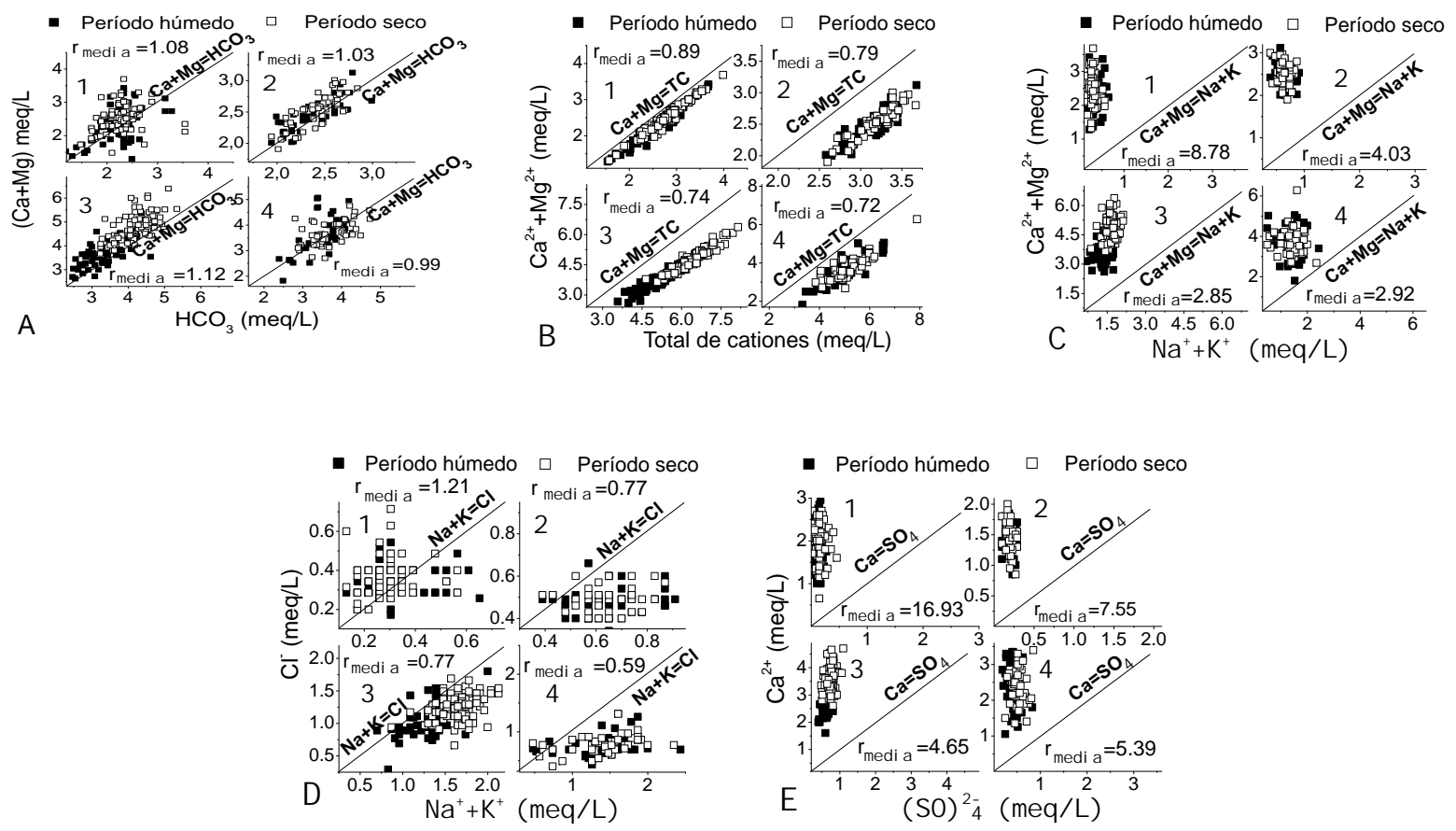

Figura 4. Relación entre las concentraciones iónicas (meq/L) en los cuatro embalses estudiados. Cada embalse se identificó con un número: Paso Bonito (1), Avilés (2), Abreus (3) y El Salto (4). La figura 4A expresa la relación entre el $\mathrm{Ca}^{2+}+\mathrm{Mg}^{2+}$ y el ( $\left.\mathrm{HCO}_{3}\right)^{-}$; $4 \mathrm{~B}$, la relación entre el $\mathrm{Ca}^{2+}+\mathrm{Mg}^{2+}$ y los iones totales; $4 \mathrm{C}$, la relación entre el $\mathrm{Ca}^{2+}+\mathrm{Mg}^{2+}$ y el $\mathrm{Na}^{+}+\mathrm{K}^{+}$; $4 \mathrm{D}$, la relación entre el Cl${ }^{-}$ y el $\mathrm{Na}^{+}+\mathrm{K}^{+}$, y $4 \mathrm{E}$, la relación entre $\mathrm{Ca}^{2+}$ y $\left(\mathrm{SO}_{4}\right)^{2-}$. Las razones entre las especies iónicas se identificaron por " $r$ " y representan el promedio de las razones durante los periodos seco y húmedo. Relationship between ionic concentrations (meq/L) in the studied reservoirs. They were identified by numbers: Paso Bonito (1), Avilés (2), Abreus (3) and El Salto (4). Figure 4A, 4B, 4C and 4D shows, respectively, the relationship between $\mathrm{Ca}^{2+}+\mathrm{Mg}^{2+}$ and: $\left(\mathrm{HCO}_{3}\right)^{-}$, total ions and $\mathrm{Na}^{+}+\mathrm{K}^{+}$while $4 \mathrm{D}$ represents the relationship between $\mathrm{Ca}^{2+}$ and $\left(\mathrm{SO}_{4}\right)^{2-}$. The ratios between the ionic specie were labeled as " $\mathrm{r}$ " and they represent the mean ratio during the dry and wet periods.

lución de silicato (Alcalinidad disolución de silicato $_{=}$ Alc.total - Alc.disolución de carbonato) se exponen en la figura 5. Los valores más altos en ambos casos corresponden a los embalses localizados en la cuenca del río Damují.

\section{Estado trófico y nutrientes}

Concentraciones de $\mathrm{Ca}^{2+}$ cercanas a $40 \mathrm{mg} / \mathrm{L} \mathrm{li-}$ mitan fuertemente las concentraciones de fósforo soluble (Margalef, 1982). Estrada (1978) propuso que la precipitación del fosfato con el $\mathrm{CaCO}_{3}$ es el principal mecanismo que controla su solubilidad en aguas duras previniendo la eutrofización. Sin embargo, los altos valores de $\mathrm{Ca}^{2+}$ en los embalses estudiados (Tabla 2) no limitaron las concentraciones de TP, lo cual se demostró en los altos valores del TSI $_{\text {medio }}$ calculado para

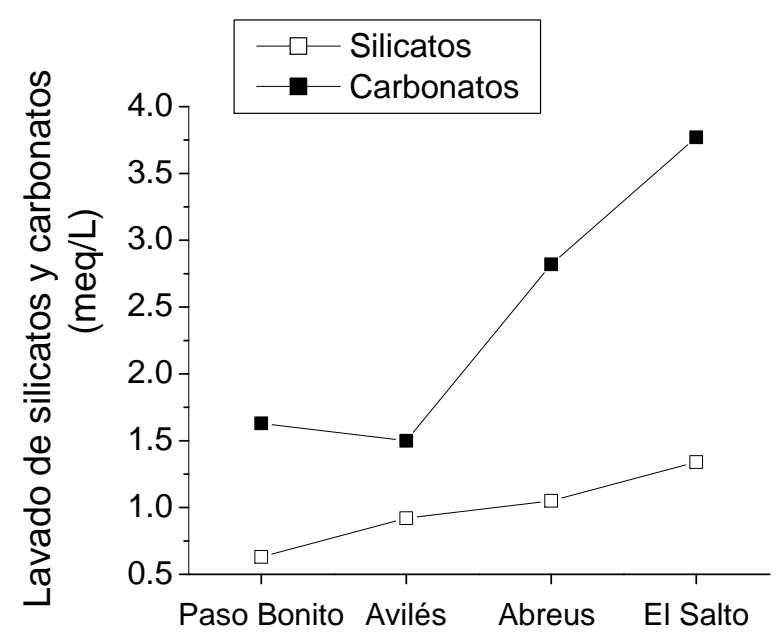

Figura 5. Contribución de la disolución de carbonatos y silicatos a la alcalinidad del agua en los cuatros embalses. The contribution of weathering of carbonates and silicates to total alkalinity in the four considered reservoirs. 

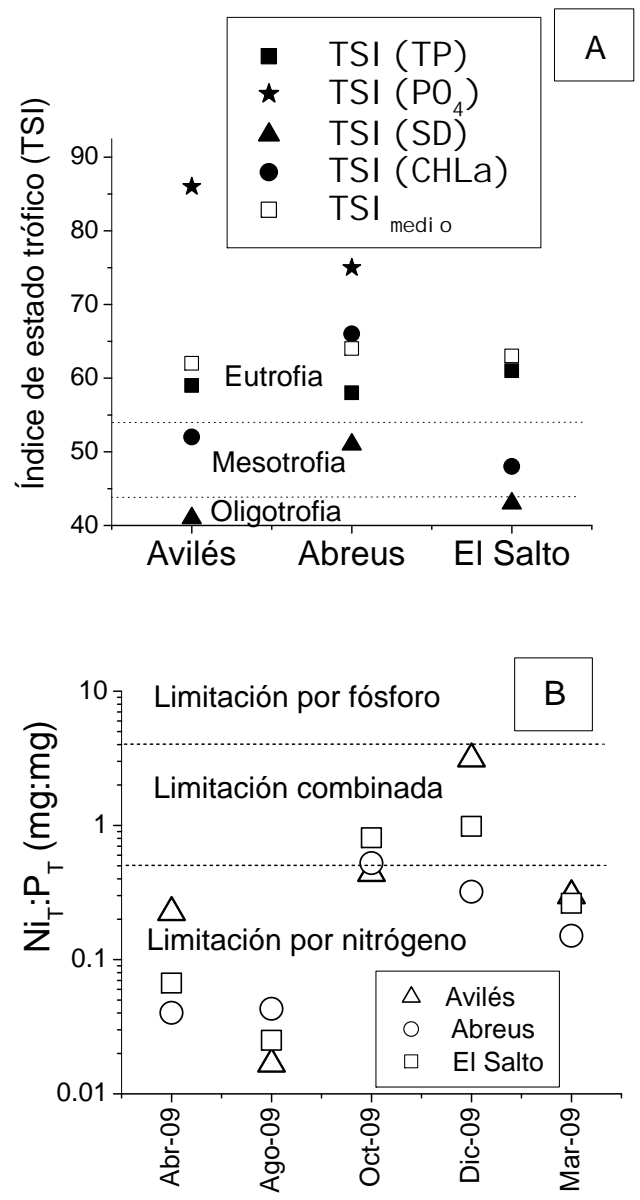

Figura 6. A: Índice del estado trófico medio de las variables $\mathrm{TP},\left(\mathrm{PO}_{4}\right)^{3-}$, SD y CHL $a$, calculado según el criterio de Carlson (1977), modificado por Toledo et al. (1983) para zonas tropicales. Se representan los índices de estado trófico calculados con las concentraciones promedio de fósforo total: TSI (TP); ortofosfato: TSI $\left(\mathrm{PO}_{4}\right)$; clorofila $a$ : TSI(CHL) y de las mediciones promedio de la transparencia: TSI(SD), así como el índice de estado trófico calculado con el promedio de los TSI de cada variable: TSI $_{\text {medio. Las líneas de puntos separan los límites }}$ del estado trófico. En 6B se representan las relaciones entre el nitrógeno inorgánico y el fósforo total. Las líneas horizontales indican niveles de la razón de $\mathrm{Ni}_{\mathrm{T}}$ y $\mathrm{TP}$ sugeridos por Morris y Lewis (1988) para identificar el nutriente limitante. A: Mean trophic state index for $\mathrm{TP},\left(\mathrm{PO}_{4}\right)^{3-}$, transparency and chlorophyll a, computed according to the criterion of Carlson (1977) modified by Toledo et al. (1983) for tropical zones. Trophic State Index calculated by means of the average concentrations of: total phosphorus: TSI(TP); orthophosphate: TSI $\left(\mathrm{PO}_{4}\right)$; chlorophyll a: TSI $(\mathrm{CHL})$ and the average measurements of transparency: TSI(SD). Additionally, the Trophic State Index calculated with the average of the TSI of each variable: TSI $I_{\text {medio }}$ is presented. The dotted line shows the limits between the different trophic states. B: relationships between inorganic nitrogen and total phosphorus. The horizontal lines indicate DIN:TP ratios suggested by Morris and Lewis (1988) to identify the limiting nutrient for phytoplankton. las concentraciones de $\mathrm{TP}$ y $\mathrm{PO}_{4}$ en los embalses Abreus, Avilés y El Salto (Fig. 6A). Estos resultados demostraron que la precipitación del fósforo es un proceso complejo y que en aguas duras también se verifican procesos de eutrofia.

El uso del TSI de Carlson (1977) modificado por Toledo y colaboradores (1983) reveló un predominio de aguas eutróficas en los cuatro embalses. De acuerdo con el TSI calculado para la CHL $a$ como variable diagnóstico, los embalses Avilés y El Salto fueron mesotróficos y Abreus fue eutrófico, mientras que el TSI calculado con los valores de la transparencia clasificó a los embalses El Salto y Avilés como oligotróficos y al embalse Abreus como mesotrófico. El TSI resultado de la aplicación de la ecuación que usa el valor medio de todos los índices (TSI modificado medio) clasificó a estos tres embalses como eutróficos (Fig. 6A).

Las correlaciones entre los TSI de las tres variables diagnóstico fueron positivas. Solo resultó significativa $(p<0.01)$ la correlación TSI(CHL) vs TSI(SD).

Los resultados de las diferencias entre los TSI para las variables diagnóstico en los tres embalses estudiados se muestran en la figura 7B. En el eje de las abscisas se representaron las diferencias entre los TSI(CHL) y TSI(SD) y en las ordenadas las diferencias entre TSI(CHL) y TSI(TP). Cuando se comparan las figuras 7A y 7B se advierte que la mayoría de las observaciones se ubicaron en el área donde predominan las partículas grandes y la herbivoría del zooplancton. Todos los puntos se ubicaron por debajo de la diagonal y separado del origen (con un intercepto en $y=-21.07$ ).

El $80 \%$ de los valores de la razón $\mathrm{Ni}_{\mathrm{T}}$ : TP fue menor que 0.5 , lo que mostró al nitrógeno como el nutriente limitante del desarrollo fitoplanctónico en los embalses Avilés, Abreus y El Salto, según la aplicación del criterio de Morris y Lewis (1988). El $20 \%$ de los valores reveló limitación por ambos nutrientes $(0.5<\mathrm{NiT}$ : $\mathrm{TP}<4)$ (Fig. 6B).

\section{DISCUSIÓN}

Las relaciones iónicas y los diagramas de Gibbs (1970) y trilineal permiten establecer cómo han 

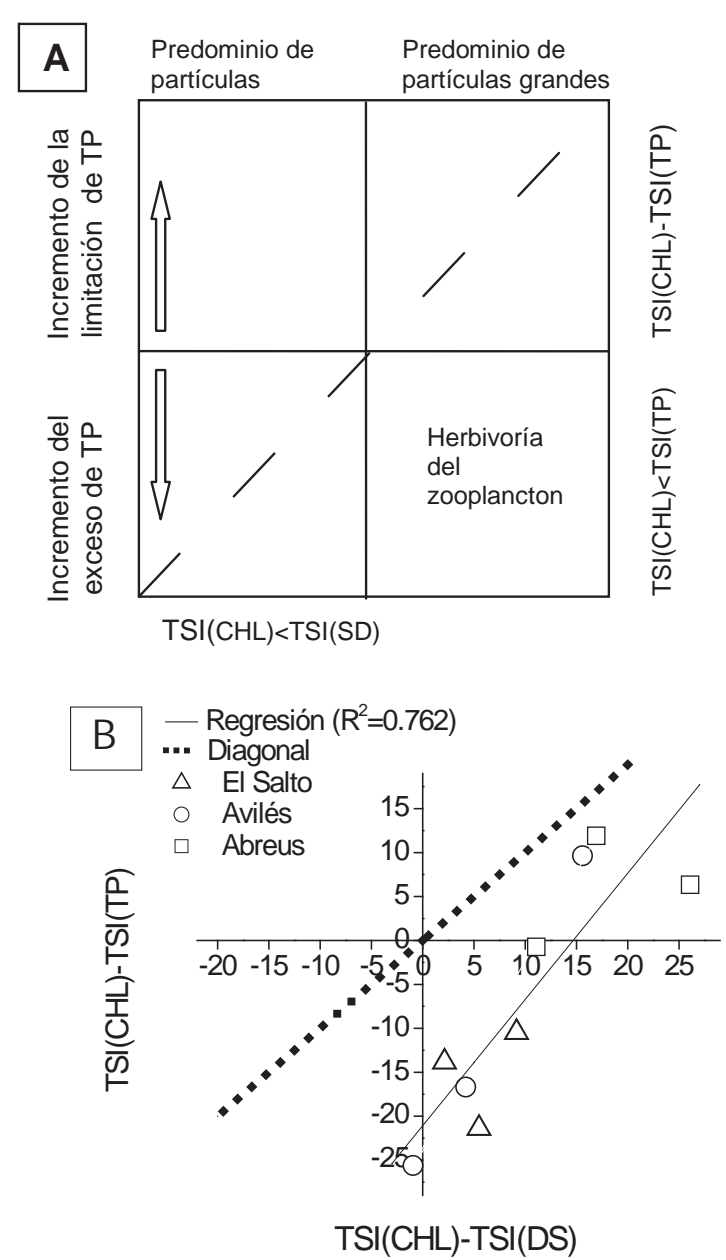

Figura 7. A: Posibles interpretaciones de las desviaciones del índice de estado trófico, según Carlson y Havens (2005). B: diferencias de los Índices de Estado Trófico para los tres embalses estudiados. La mayor cantidad de observaciones se localizaron en el cuadrante que corresponde con el predominio de partículas grandes y la herbivoría del zooplancton. A: Possible interpretations of deviations of Trophic State Index (TSI) values of Carlson 1977, according to Carlson and Havens (2005). B: differences between the trophic state index for the three studied reservoirs are showed in $7 B$. Most of the observations were located in the quadrant of the predominance of big particles and zooplankton's herbivory.

sido los procesos geoquímicos en una cuenca determinada (Fernández \& Miretzky, 2004; Pehlivan \& Yilmaz, 2005; Rajmohan \& Elango, 2007). Este estudio, realizado a partir de una base de datos de 20 años en dos cuencas con diferente localización geográfica, representa una referencia que servirá para comparar la composición química del agua y los procesos vinculados. Esta técni- ca demuestra que la meteorización de la roca es el proceso natural más influyente en la química del agua de los cuatro embalses estudiados.

Los valores más elevados de la salinidad en los embalses Abreus y El Salto están relacionados con la génesis de las rocas donde yacen las cuencas que alimentan dichos embalses, en las cuales predominan las rocas sedimentarias, que son más vulnerables a los procesos de meteorización (Bruhns \& Ramdohr, 1968). En las cuencas de Avilés y Paso Bonito, predominan las rocas ígneas que por su dureza son más resistentes a los procesos de meteorización.

La elevada proporción de $\mathrm{Ca}^{2+}$ encontrada en el embalse Paso Bonito también guarda relación con su geología. Según un estudio realizado por IGT (2008), en esta cuenca se localiza una zona cárstica, con abundantes surgencias.

La ubicación de las observaciones próximas al vértice en el diagrama trilineal (Fig. 3) y los resultados de las razones iónicas corroboraron el aporte de la disolución de carbonatos a la mineralización del agua en los cuatro embalses (Baca y Threlkeld, 2000). Para la cuenca del río Damují la disolución de los silicatos fue mayor (Fig. 5) en correspondencia con su geología. Jha et al. (2009) obtuvieron resultados similares en una cuenca caracterizada por la presencia de basaltos (silicatos) y carbonatos.

El $\mathrm{CO}_{2}$ necesario para la disolución de silicatos proviene de la atmósfera y es el doble del que se necesita para el de los carbonatos (Raymahashay, 1986; Subramanian et al., 2006). Sin embargo, la fuente para este último puede ser, además de la atmósfera, la descomposición de la biomasa presente en el suelo. Por esta razón, la disolución de los silicatos representa un importante sumidero del $\mathrm{CO}_{2}$ atmosférico y es de especial interés en el control de sus concentraciones en la atmósfera y el océano a escala de tiempo geológico (Berner et al., 1983; Brady \& Carrol, 1994). Los resultados obtenidos significaron que los embalses Abreus y El Salto contribuyeron en mayor medida en la disolución de silicatos y por tanto en la retención del $\mathrm{CO}_{2}$ atmosférico que el resto de los embalses estudiados.

Los valores de la razón $\mathrm{Cl}^{-}: \mathrm{Na}^{+}+\mathrm{K}$ inferiores a la unidad sugieren la ocurrencia de la diso- 
lución de silicatos de $\mathrm{Na}^{+}$y $\mathrm{K}^{+}$y de aporte de $\mathrm{Na}^{+}$por actividades antrópicas (Das et al., 2005; Krishnaswami \& Singh, 2005). En la cuenca de estos embalses se localizan fuentes de contaminación como son los asentamientos humanos y la cría de ganado vacuno y porcino (en mayor cuantía en los embalses Abreus y El Salto). La influencia de las actividades antrópicas sobre la calidad del agua también se reveló en los TSI obtenidos.

La localización del embalse Abreus al final de la cuenca del río Damují justifica las concentraciones significativamente inferiores de los iones mayoritarios durante el periodo húmedo. En esta etapa ocurre la dilución del agua embalsada producto de los volúmenes de agua de escorrentías que se incorporan desde la cuenca. En este embalse el área de la cuenca es 199 veces superior al área del embalse (Ac:Ae; Tabla 1), mientras que para el resto de los embalses estudiados las razones Ac:Ae son menores.

La correlación significativa entre los TSI (CHL) y TSI(SD) sugiere que la turbiedad del agua en estos embalses se debe a la CHL $a$ del fitoplancton. En los mismos se detectó un florecimiento de Microcystis spp, cianobacteria colonial (Comas et al., 2010), cuya presencia justifica la ubicación de la mayoría de las observaciones en el área correspondiente a partículas grandes y valores de TSI(CHL)-TSI(SD) $>0$.

El uso de este método gráfico también reveló que en estos embalses hay menos CHL $a$ que la predicha por las concentraciones de TP (observaciones por debajo de la línea y $=0$ ). Cuando esto ocurre significa que factores como la limitación de nitrógeno o la herbivoría del zooplancton están limitando el desarrollo del fitoplancton (Carlson \& Havens, 2005). La limitación de nitrógeno en estas aguas también fue observada al aplicar el criterio de Morris y Lewis (1988).

La mineralización de la roca determina la composición química del agua en los embalses estudiados, pero la actividad del hombre está cambiando estos sistemas. Se recomienda establecer planes de remediación encaminados a reducir los vertidos de compuestos orgánicos en las cuencas de estos embalses, con el fin de mitigar los procesos de eutrofización.

\section{BIBLIOGRAFÍA}

APHA-AWWA-WPCF. 1998. Standard methods for the examination of water and wastewater. $20 .^{\mathrm{a}} \mathrm{ed}$. Washington, DC. 1100 pp.

BACA, R. M. \& S. T. THRELKELD. 2000. Inland dissolved salt chemistry: statistical evaluation of bivariate and ternary diagram models for surface and subsurface waters. Journal of Limnology, 59(2): 156-166.

BERNER, R. A., A. C. LASAGA \& R. M. GARRELS. 1983. The carbonate-silicate geochemical cycle and its effect on atmospheric carbon dioxide over the past 100 million years. American Journal of Science, 283: 641-683.

BETANCOURT, C., R. SUÁREZ \& L. TOLEDO. 2009. Ciclo anual del nitrógeno y el fósforo en el embalse Paso Bonito, Cienfuegos, Cuba. Limnetica, 28: 79-90.

BRADY, P. V., \& S. A. CARROL. 1994. Direct effects of $\mathrm{CO} 2$ and temperature on silicate weathering: Possible implications for climate control. Geochimica et Cosmochimica Acta, 58: 1853-1856.

BRUHNS, W., \& P. RANDOHR. 1968. Petrografía, 1. ${ }^{\mathrm{a}}$ ed. Edición Revolucionaria. La Habana, 125 pp.

CARLSON, R. E. 1977. A Trophic State Index for Lakes. Limnology and Oceanography, 22: 363369.

CARLSON, R. E., \& K. E. HAVENS. 2005. Simple Graphical Methods for the Interpretation of Relationships Between Trophic State Variables. Lake and Reservoir Management, 21 (1): 107-118.

CHUNG, C. H., C. F. YOU \& H. Y. CHU. 2009. Weathering sources in the Gaoping (Kaoping) river catchments, southwestern Taiwan: Insights from major elements, $\mathrm{Sr}$ isotopes, and rare earth elements. Journal of Marine Systems, 76(4): 433443.

COMAS, A., A. MOREIRA, C. BETANCOURT \& F. JORGE. 2010. Cyanobacteria blooms in freshwater reservoir of Central Cuba. Harmful Algae News, 43: 18-19.

DAS, A., S. KRISHNASWAMI, M. M. SARIN \& K. PANDE. 2005. Chemical weathering in the Krishna Basin and Western Ghats of the Deccan Traps, India: Rates of basalt weathering and their controls. Geochimica et Cosmochimica Acta, 69(8): 2067-2084. 
DODDS, W. K. 2007. Trophic state, eutrophication and nutrient criteria in streams. Trends in Ecolology and Evolution, 22(12): 669-676.

DURFOR, C. N., \& E. BECKER. 1964. Public water supplies of the 100 largest cities in the United States, 1962. U.S. Geological Survey Water-Supply Paper 1812. 364 pp.

ESTRADA, M. 1978. Relationship among biological and physicochemical parameters in Spanish reservoirs. Verhandlungen des Internationalen Verein Limnologie, 20: 1642-1646.

FERNÁNDEZ, A., \& P. MIRETZKY. 2004. Ionic relations: a tool for studying hydrogeochemical processes in Pampean shallow lakes (Argentina). Quarternary International, 114(1): 113-121.

GIBBS, R. J. 1970. Mechanisms controlling world water chemistry. Science, 170: 1088-1090.

GONZÁLEZ, J. 2000. Guía Metodológica para el estudio integral de cuencas hidrológicas de cuencas superficiales con proyección de manejo. Grupo de Hidroclimatología y Manejo de Cuencas. Facultad de Geografía. Universidad de la Habana. 32 pp.

INSTITUTO DE GEOGRAFÍA TROPICAL (IGT). 2008. Estudio de los recursos hídricos en la cuenca del río Arimao. Proyecto Ramal, Ministerio de Ciencia Tecnología y Medio Ambiente, La Habana, Cuba. 146 pp.

JHA, P. K., J. TIWARI, U. K. SINGH, M. KUMAR \& V. SUBRAMANIAN. 2009. Chemical weathering and associated $\mathrm{CO}_{2}$ consumption in the Godavari river basin, India. Chemical Geology, 264(1-4): 364-374.

KRISHNASWAMI, S. \& S. K. SINGH. 2005. Chemical weathering in the river basins of the Himalaya, India. Current Science, 89(5): 841-849.

LI, S., Z. XU, H. A. WANG, J. WANG \& Q. ZHANG. 2009. Geochemistry of the upper Han River basin, China: 3: Anthropogenic inputs and chemical weathering to the dissolved load. Chemical Geology, 264(1-4): 89-95.

MARGALEF, R. 1983. Limnología. Editorial Omega,
Barcelona. $1010 \mathrm{pp}$.

PETER, A. 2008. Taste and Odor in Drinking Water: Sources and Mitigation Strategies. Tesis doctoral. Swiss Federal Institute of Technology Zurich. 143 pp.

PEHLIVAN, R. \& O. YILMAZ. 2005. Water quality and hydrogeochemical characteristics of the River Buyukmelen, Duzce. Turkey. Hydrological Processes, 19: 3947-3971.

PIPER, A. M. 1944. A graphic procedure the geochemical interpretation of water analysis. Eos. Transactions of the American Geophysical Union, 25: 914-923.

RAJMOHAN, N. \& L. ELANGO. 2007. Hydrogeochemistry and its relation to groundwater level fluctuation in the Palar and Cheyyar river basins, southern India. Hydrological Processes, 20, 2415-2427.

RAYMAHASHAY, B. C. 1986. Geochemistry of bicarbonate in river water. Journal of the Geological Society of India, 27: 114-118.

SÖMEK, H., M. R. USTAOĞLU \& M. YAĞCI. 2008. A Case Report: Algal Bloom of Microcystis aeruginosa in a Drinking-Water Body, Eğirdir Lake, Turkey. Turkish Journal of Fisheries and Aquatic Sciences, 8: 177-179.

SUBRAMANIAN, V. V., D. U. ITTEKKOT \& N. MADHAVAN. 2006. Silicate weathering in South Asian Tropical River Basins. In: Role of silica in Land-Sea Interactions. V. Ittekkot, D. Unger, C. Humborg, and N. Tac An (eds.): 3-13. Scope Washington D.C. USA.

TOLEDO, A. P., M. TALARICO, S. J. CHINEZ \& E. G. AGUDO. 1983. A aplicação de modelos simplificados para a avaliação de processo da eutrofização em lagos e reservatórios tropicais. XIX Congresso Interamericano de Engenharia Sanitária e Ambiental. Camboriú, 1983, 57 pp.

WILLIAMSON, C. E., J. E. SAROS, W. F. C. VINCENT \& J. P. SMOL. 2009. Lakes and reservoirs as sentinels, integrators, and regulators of climate change. Limnol. Oceanogr., 54: 2273-2282. 